\title{
A propósito de los "falsos opuestos" en Ciencias Sociales -La IAP como posibilidad para la lectura integral y crítica de la realidad-*
}

Regarding the "false opposites" in Social Sciences -the PAR as a possibility for the integral and critical reading of reality-school

Esther Gutiérrez Mora

Socióloga. Magíster en Investigación Social Interdisciplinaria.

Docente Universidad Santo Tomás (Colombia)

esthergutierrez@usantotomas.edu.co

Artículo de reflexión

Fecha de recepción: Octubre 14 de 2015 • Fecha de aprobación: Diciembre 16 de 2015

\section{RESUMEN}

El presente artículo recoge algunas reflexiones en torno a los métodos cuantitativo-cualitativo y las discusiones epistemológicas que subyacen a su puesta en escena en el mundo académico. Para ello retoma algunos elementos históricos que fueron claves en la definición de sus particularidades, así como aquellos debates que generaron una ruptura en su percepción como "falsos opuestos". A partir de esto, plantea la importancia de dinamizar una lectura integral de la realidad, donde lo cuanti-cualitativo no configura una mirada excluyente sino mutuamente constitutiva, plural y dialogante. El artículo cierra con la presentación de algunas metodologías participativas, haciendo especial énfasis en la Investigación Acción Participativa (IAP), al reconocer su avance en la integración de perspectivas (cuali-cuantitativas) para la compresión situada y crítica de la realidad social.

Palabras clave: método cualitativo, método cuantitativo, subjetivismo, objetivismo, IAP, conocimiento situado.

* Este documento fue elaborado como material de enseñanza para la clase de "Metodología de la Investigación III" del programa de Sociología de la Universidad Santo Tomás. 


\section{AbSTRaCT}

This article gathers some reflections about the quantitative-qualitative methods and the epistemological discussions that underlie its staging in the academic world. In order to do so, it retakes some historical elements that were key in the definition of its peculiarities, as well as those debates that generated a rupture in its perception like "false opposites". From this, it raises the importance of dynamizing an integral reading of reality, where quantitativequalitative does not constitute an exclusive, but mutually constitutive, plural and dialogic view. The article closes with the presentation of some participatory methodologies, with special emphasis on PAR, recognizing their progress in the integration of perspectives (qualitative and quantitative) for the situated compression and critique of social reality.

Keywords: Qualitative method, quantitative method, subjectivism, objectivism, PAR, situaded knowledge.

\section{INTRODUCCIÓN}

Promover y dinamizar la investigación en Ciencias Sociales constituye un reto fundamental tanto al interior como fuera de los espacios académicos, pues representa la puerta de entrada para la compresión de problemas sociales que con el paso del tiempo, evidencian mayor grado de complejidad y la presencia de grupos poblacionales más diversos en términos culturales, políticos y económicos. En ese sentido, se plantea la urgencia de abordar dichos problemas a partir de una lectura integral y situada de la realidad social, donde lo subjetivo y objetivo no se presentan como posturas irreconciliables, sino como plurales y dialogantes.

De acuerdo a ello, abordar las "técnicas de investigación social", en tanto estrategias para la aproximación a contextos y sujetos sociales, supone una reflexión previa sobre su dimensión epistemológica y la pertinencia de su aplicación atendiendo a las particularidades de "aquello que interesa investigar", esto último teniendo en cuenta que la reflexión epistemológica es fundamental, pero el proceso de investigación no puede ser definido por la adhesión a una tradición teórica o metodológica, sino en 
términos de lo que se quiere investigar (Mejía, 2003, p. 1), en otras palabras, a partir del problema y las preguntas formuladas por el investigador.

Lo anterior tiene sentido al considerar que en investigación social aún se evidencia la selección de técnicas de investigación sin considerar las particularidades de los contextos, con prejuicios valorativos asociados a las tradiciones epistemológicas de donde surgen, ejemplo de ello el positivismo, que en su momento legitimó el diseño de la encuesta cerrada como uno de los instrumentos por excelencia para llevar a cabo una aproximación objetiva de la realidad, y del lado opuesto las perspectivas fenomenológicas como única posibilidad para una aproximación real a las dinámicas y cotidianidad de los sujetos sociales.

El presente artículo profundiza en la importancia que tiene el abordaje de la realidad social desde una perspectiva integracionista y situada, aterrizando en alternativas metodológicas como la IAP, la cual se caracteriza por estar abierta a reelaboraciones y por supuesto a la creación de nuevas formas de aproximación.

\section{EL DEBATE EPISTEMOLÓGICO COMO PUNTO DE PARTIDA}

La discusión en torno al problema del conocimiento, sea este empírico o teórico, ha estado determinada por las apuestas metodológicas y epistemológicas que orientan al investigador. Su condición de sujeto social, aunada a la de científico e investigador, ha evidenciado históricamente una tendencia hacia la toma de posición frente a dos alternativas aparentemente irreconciliables, por un lado la posición subjetivista que se impone al reconocer en la interacción de los sujetos sus "percepciones" como principio y fin del mundo social, contrario a aquella mirada objetivista que se queda prendada del “orden de las estructuras”, sin cuestionar qué pasa con el orden práctico.

De acuerdo a lo anterior, es importante señalar que el mayor obstáculo que se presentó a esta disputa histórica, fue encontrar una base lo suficientemente sólida desde donde las diferencias pudieran establecerse racionalmente, trascendiendo los intereses personales de los grupos de investigadores adscritos a "una u otra causa" (Tezanos, 1998, p. 11); asímismo, dichas diferencias se trasladaron al plano de la 
elección y uso de técnicas e instrumentos de recolección de información, haciendo excluyente su implementación dependiento del enfoque epistemológico privilegiado por el investigador. A este respecto, Tezanos (1998) plantea:

Las preguntas que surgieron desde el ámbito de lo epistemológico trascendieron tanto los problemas de validez y confiabilidad con que lo cuantitativo interpelaba a lo cualitativo, como la crítica a la falta de rigurosidad en los procedimientos para la recolección de datos a través de los registros observacionales y las entrevistas que impedían el recurrir a instrumentos estadísticos que abrieran el camino a la generalización de los hallazgos, que se formularon también desde lo cuantitativo (p. 20).

Con el propósito de posibilitar una comprensión más amplia de la discusión dada en torno a la relación "sujeto-objeto, sujeto-sujeto" se retomarán a manera de síntesis algunos aspectos relevantes relacionados con las corrientes epistemológicas que han liderado el debate, haciendo claridad que no se abordarán exhaustivamente las posturas que subyacen a cada método.

Es conveniente entonces abordar en un primer momento la concepción positivista. Esta nace en un contexto en el que las ciencias naturales alcanzan su máximo desarrollo y prestigio (siglo XIX), por tanto, los avances de la física, la química y la biología evidenciaron que la aplicación de sus procedimientos garantizaría el conocimiento verdadero, por ello, esta corriente epistemológica otorgará una valoración importante a los procedimientos y técnicas investigativas asociadas a los avances liderados por las ciencias naturales. Allí la sociedad es vista como una realidad objetiva independiente de los individuos, medible y cuantificable. Por tanto, se da prelación al objeto como fenómeno observable y legítimo, en tanto su aproximación se encuentre mediada por el método científico. Lo anterior da forma al paradigma explicativo en Ciencias Sociales.

Desde una perspectiva Durkheimiana, las miradas de corte positivista suponen la legitimación dentro del diseńo, de la búsqueda de causas o hechos, del empleo de la estadística como recurso lógico y operativo, de la encuesta y la observación controlada a través del experimento, como instrumentos básicos (aunque no únicos) del trabajo científico (Sandoval, 2002, p. 31). 
Frente a esta postura, surgen críticas y cuestionamientos liderados principalmente por filósofos y pensadores como Dilthey, Weber y Husserl, quienes enfrentan el debate a través de las propuestas fenomenológica y hermenéutica. Con ellos (así como con Heiddeger, Schutz, Berger, Luckman), se plantea la defensa del carácter específico de la realidad humana, donde se propone como alternativas para el análisis las categorías de sujeto, subjetividad y significación; en ese sentido, interesará desarrollar aquello que en las percepciones, sentimientos y acciones de los actores sociales aparece como pertinente y significativo, configurando entonces, el paradigma histórico-hermenéutico.

Ahora, un tercer paradigma de investigación, el critico-social, se afianza en una perspectiva dialéctico-materialista, en ella la realidad es concebida como múltiple, divergente, construida e históricamente mediada por la producción y reproducción de las relaciones humanas. Desde esta mirada, inspirada principalmente por el materialismo histórico de Marx, interesa no el objeto ni el sujeto de conocimiento, sino la interacción entre estos en contextos históricos y culturales determinados (Pulido, Ballén y Zúñiga, 2007, p. 24).

Es importante señalar que con los paradigmas hermenéutico y crítico social, se avanza en algunos puntos de mediación frente a la interacción de las formas de conocimiento de la realidad, ejemplo de ello Max Weber:

Desde una teoría de la acción, intenta hacer un planteamiento que, sin rechazar la búsqueda positivista de las explicaciones causales, sitúa el concepto de la acción individual significativa en el centro de su teoría de la sociedad (...), en ese sentido la sociología es concebida como una ciencia que aspira a la comprensión interpretativa de la acción social, para obtener la explicación causal de su curso y efectos (Sandoval, 2002, p. 32).

Los antagonismos empiezan a diluirse al reconocer que cualquier dato identificado implica tanto cualidad como cantidad, sin embargo, cuando la discusión se concentra en el ámbito de lo epistemológico, las diferencias por supuesto son irreconciliables, por ello, es en el plano de la práctica donde la perspectiva “integracionista” planteada por Eduardo Bericat (1998) debe abrirse paso, entendida no como una mezcla de métodos, sino como la posibilidad para combinar las estrategias a partir de las cuales 
se lleva a cabo el tratamiento de los datos (Mejía, 2003, p. 24). En ese contexto, hablar de estrategias, supone considerar las formas en que se obtiene, organiza, sistematiza y analiza la información.

Pierre Bourdieu también dará sustento a la ruptura de perspectivas, al expresar que el sociólogo se enfrenta permanentemente al dualismo metodológico, siendo imperativo superar los falsos opuestos "subjetivismo y objetivismo". El primero de ellos, entendido como aquel que toma las representaciones sociales como su base, por tanto la realidad se constituye en la suma de diferentes actos de interpretación a partir de los cuales la sociedad construye procesos de significación en contextos de interacción (Bourdieu y Wacquant, 1998, p. 17).

En el segundo caso (el objetivismo), la sociología se caracteriza por seguir las perspectivas positivistas y el planteamiento de Durkheim al tratar los hechos sociales como "cosas", esto con el propósito de develar el sistema de relaciones que determinan la conducta y las representaciones de los individuos (Bourdieu y Wacquant, 1998, p. 17), es decir, concentrar la mirada en las estructuras ocultas que condicionan a los sujetos sociales.

Para este sociólogo francés, constituye un imperativo generar una ruptura con esta mirada antinómica y mutuamente excluyente entre lo objetivo y subjetivo, dado que los dos posibilitan una mirada integral y complementaria de los agentes sociales y las estructuras en las que están inmersos.

Ahora, en el marco de esta misma reflexión, Bonilla y Rodríguez (1997) plantean la triangulación metodológica como alternativa clave para la articulación de perspectivas y técnicas de investigación, esto con el fin de visibilizar los diferentes aspectos de la realidad estudiada. Para ello también será fundamental el examen de lo que han denominado como "lógica reconstruida" (enfoque metodológico seleccionado), a la luz de las "lógicas en uso" (características reales de las prácticas de los científicos durante el proceso investigativo). Esto último representa un reto, en tanto las disputas por el poder y la legitimidad en los escenarios académicos, privilegian la lógica reconstruida, desconociendo en ocasiones la importancia del 
“proceso" de construcción de conocimiento como el espacio donde se juegan la destreza, capacidad reflexiva y creadora de quien investiga.

De acuerdo a lo anteriormente expuesto, puede anotarse que han sido innumerables y reiterativas las producciones intelectuales que plantean el problema del método científico y el tipo de investigación como procesos al interior de los cuales la dimensión objetiva y subjetiva de los sujetos-objetos de indagación, debe ser conciliada en la medida en que conforman una totalidad compleja e indeterminada.

Entonces, establecer una ruta efectivayadecuada paralograraquellacomplementariedad entre lo subjetivo y objetivo, dependerá de la posibilidad de ampliar los niveles de comprensión y teorización de lo social, esto último no implica dar prelación a la dimensión teórica, sino construir nuevas lecturas de la realidad a partir de un ejercicio comprensivo que se piense en la totalidad del conocimiento, tanto en su dimensión práctica como teórica, de ahí que los aportes como investigadores sociales deben superar la "descripción", en la que los datos permanecen sujetos a su situación de origen -es decir, de donde fueron tomados-, y lograr que los referentes teóricos sean tomados como herramientas para llevar a otros planos la interpretación de una realidad que es cambiante y abierta a lo indeterminado.

\section{LA POTENCIALIDAD DE LAS PERSPECTIVAS PARTICIPATIVAS}

La investigación cualitativa, a través de la incorporación de la dimensión subjetiva en la investigación social, ha puesto a disposición de quien investiga la posibilidad de generar nuevas representaciones del "sujeto-objeto" a través de opciones diferentes a las que sustentan el paradigma positivista en Ciencias Sociales. De acuerdo a ello, enfoques teóricos fundamentales de la perspectiva cualitativa, como "la etnometodología" y enfoques participativos como la "investigación-acción participativa", "la sistematización de experiencias", entre otras, conservaron en sus inicios buena parte de la ruta metodológica manifiesta en el esquema positivista de indagación. La búsqueda de algunos cánones de neutralidad y objetividad, lleva a un distanciamiento -aún manifiesto- entre lo que se considera "objeto" investigado frente al "sujeto" investigador, un distanciamiento que en términos de la relación 
saber-poder, margina la potencialidad del sujeto social investigado en aras de conservar ciertos elementos de legitimidad científica.

La crisis del positivismo se evidenció claramente en los escenarios latinoamericanos de los ańos 60 y 80; el contexto de dictadura en algunos países latinoamericanos y el debilitamiento de la estructura económica y social, contribuyó a que los investigadores sociales construyeran nuevas formas de cuestionar y acercarse a la realidad, comprometiendo de manera crítica a los sujetos investigados con la recuperación de su protagonismo como agentes de cambio en una sociedad excluyente. Es así como la IAP, la Recuperación colectiva de la historia y la sistematización de experiencias, al tener como presupuesto la puesta en marcha de un ejercicio pedagógico, dialógico y crítico con los "otros", transforma la relación "saber-poder" hasta entonces hegemónica y marginal; ahora, sujeto investigado y sujeto investigador establecen una relación entre iguales en la que construyen conjuntamente conocimiento y transforman el sentido de su práctica.

En ese contexto, la relación sujeto-sujeto que orientará la investigación social, reactiva la discusión sobre los vínculos entre teoría y práctica, dos dimensiones mutuamente constitutivas en la medida en que la construcción teórica se encuentra alimentada de los elementos que integran la realidad social y esta última a su vez, tiene la posibilidad de ser leída e interpretada desde otras perspectivas.

Lo significativo de los nuevos procesos de construcción teórica es el reconocimiento del dinamismo y reflexibidad de los sujetos; dinamismo en tanto el sujeto es móvil y está múltiplemente situado (el conocimiento deja de ser concebido como algo estático y estable) y reflexivo, pues quien investiga hace parte de la realidad, un espacio que mediado por la investigación, permite el encuentro de reflexividades, procesos de subjetivación tanto del investigador como del investigado (Marcus, 2001).

Las perspectivas cualitativas de carácter participativo, han hecho una ruptura en la investigación social, pues reflexionar la práctica presente para orientar y potenciar la práctica futura, deja entrever que la relación con el conocimiento y el poder da paso a una relación de carácter pedagógico, donde tanto investigado como investigador establecen un compromiso que va más allá de ampliar el horizonte del conocimiento, 
para posibilitar un compromiso de interpretación de la práctica del “otro", quien junto al investigador se constituye en sujeto protagonista y responsable de su devenir histórico.

La potencialidad de las perspectivas investigativas participativas radica en la posibilidad de encuentro entre subjetividades, proceso al cual se da paso al romper el muro de la objetividad científica, lo cual no implica negar el que dichas perspectivas exijan un rigor en el uso de sus técnicas e interpretaciones en el proceso investigativo, más bien, supone una forma de ubicarlas dentro de un esquema menos rígido e instrumental, donde teoría y práctica hacen parte de una construcción social llevada a cabo por los sujetos sociales.

Apostarle a estos ejercicios investigativos, implica no solo ampliar los niveles de comprensión y construcción teórica de lo social, también supone hacer una lectura crítica de la "realidad", esta última, vista no solo desde el contexto cotidiano e investigativo que problematizamos y vivimos, sino también desde el contexto de la academia y la comunidad científica de la que hacemos parte.

La academia es un espacio en el que se mueven constantemente las disputas por la legitimidad del conocimiento, por ello argumentar la potencialidad y legitimidad de perspectivas cualitativas participativas como las mencionadas en líneas anteriores, implica coherencia como investigadores con las apuestas prácticas y teóricas con las cuales construimos y reconstruimos conocimiento y realidad, es decir, dar razón de la complementariedad y mutua constitución de la teoría y la práctica, pues a ellas subyace un sujeto que, a través de su acción y conocimiento, las recrea como dimensiones del saber; su legitimación supone aceptar que tanto práctica como teoría han sido objeto de una "falsa oposición", que solo quien investiga coherente, crítica y reflexivamente puede entonces "deslegitimar".

Las apuestas teóricas que se han suscitado al intentar reconocer el proceso de constitución de los sujetos y la construcción de subjetividad, están ligadas a la discusión que se genera en torno al fracaso del proyecto emancipatorio que proclamó la modernidad, pues allí se evidenció que la subjetividad colectiva no se agotaba en lo racional ni en lo ideológico, como lo plantearon en su momento las teorías 
clásicas, tampoco que los procesos de reconocimiento y representación de las identidades se dan solo por vía de la conciencia y la razón ilustrada, por el contrario, la heterogeneidad cultural de los individuos, la indeterminación y complejidad de la realidad que construyen, planteó como desafío pensar esta última -la realidad- de forma abierta, más allá de los límites organizados de la teoría.

En ese orden de ideas, sociólogos como Giddens (1994) y Touraine (2000) han planteado la importancia de repensar y replantear los problemas y principios de la modernidad, en aras de transformar su concepción negativa y reconocer que la historia no tiene un sentido lineal, implica siempre rupturas y continuidades, crítica y reafirmación del pasado, cuestión que supone, en el caso de Touraine, dar una mirada al tránsito que existe entre "individuo", "actor" y "sujeto", apelando a la importancia de superar el narcisismo del primero, la simplicidad en el ejercicio de un rol o papel en el segundo, para dar un paso hacia el sujeto que cuestiona y transforma activamente su realidad. De acuerdo a ello será clave considerar que:

El pensamiento solo es moderno cuando renuncia a la idea de un orden general, a la vez natural y cultural del mundo, cuando combina determinismo y libertad, innato y adquirido, naturaleza y sujeto. Lo cual debe llevar a reconocer la diferencia esencial entre ciencias de la naturaleza y conocimiento social, a condición de no olvidar que existen ciencias naturales del hombre, siendo el ser humano al mismo tiempo naturaleza y sujeto (Touraine, 2000, p. 278).

\section{EL LUGAR DE LA IAP EN LA CONSTRUCCIÓN DE CONOCIMIENTO SITUADO Y CRÍTICO}

La pregunta en torno a "cómo investigar" ha sido fundamental en el proceso histórico de construcción de conocimiento; el deseo de abandonar la distancia e instrumentalidad con la cual debía ser observada la realidad social para lograr mayores niveles de objetividad - una de las exigencias de la escuela Positivista-, se convirtió en motivación para la construcción de metodologías que causarían una ruptura epistemológica con los presupuestos teóricos y metodológicos hasta entonces hegemónicos -los supuestos empiristas y positivistas-. 
La crítica a las formas de conocimiento tradicional, aunada a la complejidad con la cual se hacían manifiestas la diferentes problemáticas sociales, establecieron las bases para el surgimiento de la propuesta de IAP, sin embargo, es importante aclarar que las versiones sobre su aplicación varían según el autor y la región de la cual emerge, ejemplo de ello, Kurt Lewin inspira y presenta algunos elementos esenciales relacionados con la IAP, otros investigadores la reformulan y adaptan a las exigencias y especificidades de cada contexto, es el caso de Paulo Freire y Orlando Fals Borda principalmente en América Latina.

La metodología de la IAP, se ha desarrollado y aplicado en proyectos sociales de diversa índole, que buscan crear modelos alternativos de planificación, ejecución y evaluación de procesos de transformación social, dinamizando la participación colectiva en la investigación y en la acción de los diversos sectores de la sociedad, estos últimos considerados años atrás, solo como objetos pasivos de estudio (Salazar, 1992, p. 10).

Esto varía -como se enunció en líneas anteriores- según el contexto y los autores, por ello para hablar de los inicios de esta propuesta metodológica es fundamental dar una mirada a los escenarios de los cuales emergen las dos tradiciones contemporáneas más destacadas: "la anglosajona" y la "latinoamericana".

En la tradición anglosajona se destaca principalmente el norteamericano Kurt Lewin, quien según Pulido (2002):

Es el creador de la línea de investigación social ligada a la acción trasformadora, ya que los principios orientadores de la "investigación-acción" -o actionresearch- aparecen en su obra en el ańo de 1944, algunos de ellos son: el carácter participativo, el impulso democrático y la contribución al cambio social (pp. 34-35).

Ahora, en el contexto latinoamericano se destacan dos vertientes de gran significación para la consolidación de la corriente de la IAP, por un lado, la vertiente educativa desarrollada por el brasileño Paulo Freire, quien con su propuesta de “investigación 
temática" (Torres, 1995, p. 33) reacciona contra el positivismo pedagógico, incentivando la participación de los trabajadores y sectores populares en la gestión de su propia educación. Por otro lado, la vertiente sociológica desarrollada principalmente por el colombiano Orlando Fals Borda, quien a través de su separación con el positivismo y el funcionalismo presentes en la investigación social, promueve en un primer momento,el estudio activo de la situación de la población rural, considerada víctima principal de los sistemas dominantes y las políticas de desarrollo vigentes en su contexto (finales de los años 50 a 60).

La IAP surge a partir del dinamismo y las condiciones sociopolíticas de los años 60, ejemplo de ello, la transformación social desatada por la revolución cubana y el proceso de cambio reformista promovido por la Alianza para el Progreso. Lo proyectos de Reforma Agraria, son articulados a la propuesta de IAP, siendo sus principales beneficiarios obreros, campesinos y pueblos indígenas, sujetos que a través de la educación popular concientizadora, lograrían promover procesos emancipatorios de cambio social radical.

A finales de los años 70 , se produce un desplazamiento en la orientación y actores de la IAP, pues se focaliza en la organización del sector urbano-marginal, dejando atrás al campesino. Este desplazamiento acompañó el proceso de democratización político y social en América Latina que provocó una redefinición en el enfoque de las estrategias de investigación. Durante este periodo, los investigadores se concentraron en el análisis de problemas sociales, como los niveles críticos de pobreza -los cuales estaban aumentando en las áreas urbanas a un ritmo más acelerado que en las áreas rurales-, la marginalidad y el desempleo crecientes en la economía formal, fenómenos que respondían a las presiones de la deuda externa y la crisis fiscal de Estado.

Para Carlos Torres, Paulo Freire y Orlando Fals Borda, coincidieron en su reflexión en torno a la forma en que el proceso creciente de marginación que ha existido en el sector público, constituía una razón más para que los proyectos de educación

1 El objeto de la investigación temática propuesta por Freire, es encontrar los temas generativos o las expresiones de la palabra del pueblo, con el propósito de facilitar la programación educativa y el desarrollo concientizador de la acción cultural. 
popular a los cuales apunta la IAP, tuviesen como uno de sus objetivos fundamentales promover la concientización y la organización política de los sectores de la población menos favorecidos (Torres, 1995, p. 42). Esto se lograría en la medida en que los actores sociales reconocieran su condición de sujetos históricos y lograran potenciar su acción crítica.

La IAP en América Latina ha sido inspirada por la propuesta pedagógica de Paulo Freire, sin embargo, buena parte de su plataforma ideológica y metodológica está ligada, además de algunos postulados de Marx -la dialéctica-, al concepto de “intelectual orgánico” desarrollado por Antonio Gramsci. A través de esta categoría, los investigadores latinoamericanos que le apostaron al desarrollo de la IAP lograron comprender que:

Para que los agentes externos se incorporaran en una vanguardia orgánica, debían establecer con el pueblo una relación horizontal -una relación verdaderamente dialógica sin presunción de conciencia avanzada-, involucrarse en las luchas populares y estar dispuestos a modificar las propias concepciones ideológicas mediante una interacción con estas luchas; los líderes orgánicos deberían estar dispuestos a rendir cuentas a los grupos de base en todas las formas genuinamente democráticas y participativas (Fals, 1992, p. 209).

A estas dos líneas de pensamiento se suma el punto de vista crítico de los filósofos y científicos sociales de la Escuela de Frankfurt -Adorno, Marcuse, Habermas-, quienes plantean, según Kemmis (1992):

Que la gran influencia del positivismo resultó en un amplio crecimiento de la racionalidad instrumental y en la tendencia a observar todos los problemas prácticos como asuntos técnicos. Los miembros de la Escuela, buscaban articular el punto de vista teórico, cuya tarea central era la emancipación tanto de los investigadores como de los grupos investigados, así como la compresión de lo significativo de su acción frente a la dominación del pensamiento positivista. El reto entonces, se centró en reactivar la capacidad de reflexión del individuo sobre sus propias situaciones e incentivar un cambio mediante su acción (pp. 182-183). 
Los puntos de vista dialéctico, fenomenológico y hermenéutico ofrecieron elementos epistemológicos para la investigación y la práctica en educación que buscaba tomar distancia de los supuestos positivistas y empiristas que hacían parte de los enfoques convencionales en investigación social.

La IAP compartirá el compromiso al que convoca el "intelectual orgánico" de Gramsci y comprenderá el "conocimiento" como un instrumento de transformación social que requiere de su constante interacción con la praxis, relación a la cual apeló permanentemente la Escuela de Frankfurt (su crítica al positivismo y a la investigación instrumental deja entrever solo algunos elementos de los cuales se nutre a nivel epistemológico esta perspectiva investigativa).

Es así como los lineamientos de la IAP, al partir de la teoría crítica de la Escuela de Frankfurt, rechazan las ya nombradas nociones positivistas de racionalidad, objetividad y verdad, cuestionando el abordaje de la realidad desde algunas categorías básicas y específicas del conocimiento formuladas en su mayoría desde los modelos presentados por la Ciencias Naturales. De esta manera, la propuesta participativa de la investigación-acción acepta las categorías interpretativas de los sujetos-objetos investigados, orientando la práctica hacia el análisis y la transformación de la realidad investigada.

Con lo anterior, puede anotarse que el proceso emancipatorio que subyace a la propuesta de la IAP, toma mayor fuerza en el continente latinoamericano, convirtiéndose en el escenario por excelencia para la aplicación de metodologías participativas, pues su condición histórica de dependencia y desigualdad social hace que las investigaciones y sus investigadores le apuesten a la construcción de un conocimiento más humanizante, donde el "otro" -investigado- tenga la posibilidad de reconocerse "en" el mundo y "con" el mundo, abandonando su condición de actor pasivo para dar un salto a la condición de sujeto crítico y participativo.

Es así como los problemas de nuestro contexto obligan a que la concepción de ciencia y metodología que se presenta como hegemónica y totalizante -tradición científica Occidental- sea repensada a partir de la complejidad y singularidad de nuestro entorno, luego esto no implica, negar la tradición que en términos teóricos y 
metodológicos alimentan buena parte de la producciones que le dieron su andamiaje a las ciencias sociales, sino generar reflexiones y acciones con "sentido" frente a lo singular de nuestros actores y escenarios.

La puesta en escena de la propuesta de la IAP evidencia ese primer intento reflexivo (sujeto investigado, sujeto investigador) de construcción de conocimiento y conciencia crítica, que a través de un ejercicio pedagógico, busca incentivar la participación de los sujetos sociales en la transformación de las estructuras sociales dominantes y hegemónicas.

En ese sentido, puede anotarse que las perspectivas participativas no solo se presentan a la investigación social en América Latina como posibilidad dialógica e incluyente de los sujetos sociales y sus contextos, sino también constituyen una oportunidad para articular críticamente perspectivas metodológicas que permiten una comprensión situada e integral de la realidad social, siendo un reto impostergable entenderlas como miradas mutuamente constitutivas y no excluyentes. En palabras de Trujillo (2013) citando a Haraway (1995):

(...) Los conocimientos situados implican responsabilidad, es decir, un posicionamiento crítico, en otras palabras, objetividad. Implica también ponerse en el lugar del otro, mirar desde abajo, con otros ojos a ese otro, "como actor y como agente, no como una pantalla o un terreno, no un recurso, nunca como esclavo del amo que cierra la dialéctica en su autoría del conocimiento objetivo" (Haraway, 1995), implica pues una relación social diferente, ya no de dominación, en el que es posible mirar y también ser mirado (p. 7).

\section{A MODO DE CONCLUSIÓN}

La mirada integracionista es un imperativo en la investigación social, pero esta apuesta requiere no solo de una conciencia clara frente al camino que históricamente ha transitado este debate, también es fundamental que la ruptura de los "falsos opuestos” y el posicionamiento de metodologías participativas localizadas se dé 
desde las instancias universitarias y se traduzca en planes de estudios y programas académicos en Ciencias Sociales, que evidencien una transformación sustancial del currículo.

Si bien en el imaginario de quienes hacemos parte de las Ciencias Sociales en América Latina, el debate "objetivo-subjetivo", "cantidad-cualidad" es bien conocido y aparentemente superado, resulta paradójico encontrar programas de estudio en Ciencias Sociales o disciplinas de las Ciencias Sociales, que continúan privilegiando en la distribución de sus créditos académicos la enseńanza de las teorías por encima de aquellos espacios académicos (o asignaturas) que tienen como propósito el abordaje de las metodologías o la praxis investigativa.

Aun en el inconsciente académico se considera que el estatuto de cientificidad y la identidad disciplinar están dadas por el objetivismo teórico y la enseñanza de técnicas de investigación que posibilitan la medición y la comprobación. Esta situación no solo evidencia el desconocimiento de los avances históricos que tuvo el debate anteriormente expuesto, sino también la incidencia particular que tiene en América Latina la formación de profesionales en Ciencias Sociales e investigadores que se aproximan a la realidad sin una mirada situada y localizada.

La reflexividad crítica es producto de la posibilidad de anclar el conocimiento a los contextos que queremos abordar, también del estar abiertos al enriquecimiento de propuestas metodológicas participativas con perspectivas situadas. Es por ello que resalto en la presentación de este texto la IAP, en tanto constituye un punto de partida interesante para poner en discusión el compromiso y la incidencia de la investigación en la transformación social. Dicha transformación también pasa por la concepción de lo que se considera es o no es científico y por ende se traduce en programas de formación en investigación y en Ciencias Sociales que promueven la generación de un conocimiento que no solo busca adaptar sus prácticas a modelos y estándares globales de medición de calidad, sino también se da el permiso de generar puntos de fuga e innovaciones que aceptan el conocimiento propio como parte de la configuración de lo disciplinar y de los diferentes campos del saber: 
(...) Lo que está en juego no es solo la posibilidad de disputar los contenidos hegemónicos (que ya es bastante), sino también, las formas de pensar, las maneras de producir conocimiento riguroso, los lenguajes y las comunicaciones. (...) No se trata, por supuesto, del desprecio inconducente a los enormes aportes de corrientes de pensamiento universales, pero sí de abandonar el absurdo de intentar encastrar procesos emergentes en lo dado, como si todo estuviera dicho, como si pudieran aplicarse las teorías, como si no existiera lugar para lo que está siendo y, aun, para lo inédito (Retamozo, Morris y Bracco, 2014, pp. 62-63). 


\section{ReFerenCIAS}

Bericat, E. (1998). La integración de los métodos cualitativo y cuantitativo en la investigación social. Significado y medida. Barcelona, España: Ariel.

Bonilla-Castro, E., y Rodríguez, P. (1997). La investigación en Ciencias Sociales. Más allá de del dilema de los métodos. Bogotá: Grupo Editorial Norma.

Bourdieu, P. y Wacquant, L. (1998). Respuestas por una antropología reflexiva. México: Grijalbo.

Fals, O. (1992). La investigación acción participativa. Inicios y desarrollos. La situación actual y las perspectivas de la investigación-acción participante en el mundo. Bogotá: Magisterio.

Giddens, A. (1994). Consecuencias de la Modernidad. Madrid: Alianza Editorial.

Haraway, D. (1995). Conocimientos situados. Ciencia, cyborgs y mujeres. Madrid: Cátedra.

Kemmis, S. (1992). Mejorando la educación mediante la investigación acción. En La investigcación acción participativa. inicios y desarrollos. Bogotá: Magisterio.

Marcus, G. (2001). Etnografía en/del sistema mundo. El surgimiento de la etnografía multilocal. Alteridades, 11(22), 111-127.

Mejía, R. (2003). Combinación estratégica: investigación sociocultural cualitativa-cuantitativa. S. d. S. A. (Ed.). Nómadas, 18, 20-27.

Pulido, R. (2002). Abordaje hermenéutico de la investigación cualitativa. Bogotá: Ediciones Grancolombianas.

Pulido, R., Ballén, M. y Zúñiga, F. (2007). Abordaje hermenéutico de la investigación cualitativa. Teorías, proceso, técnicas. Bogotá, Colombia: Universidad Cooperativa de Colombia. 
Retamozo, M., Morris, B. y Bracco, O. (2014). Los desafios de las Ciencias Sociales y el pensamiento politico en América Latina (algunas notas). Argentina: Universidad Nacional de la Plata. Oficios Terrestres (n. ${ }^{\circ}$ 31). Recuperado de http://perio.unlp.edu.ar/catedras/ system/files/retamoso_y_otros_-_los_desafios_de_las_cs_sociales_en_al.pdf

Salazar, M. C. (1992). La investigación acción participativa. Inicios y desarrollos. Bogotá, Colombia: Magisterio.

Sandoval, C. (2002). Investigación cualitativa. I. C. ICFES. (Ed.). Bogotá, Colombia: ARFO Editores e impresores Ltda.

Tezanos, A. D. (1998). Una etnografia de la etnografia: aproximaciones metodológicas para la enseñanza del enfoque cualitativo-interpretativo para la investigación social. Bogotá: Antropos.

Torres, C. (1995). Estudios freireanos. Montevideo: Editorial Libros del Quirquincho.

Touraine, A. (2000). Crítica de la modernidad. México: Fondo de Cultura Económica.

Trujillo, C. (2013). Epistemologías otras en la investigación social, subjetividades en cuestión. CLACSO, (23). Red de posgrados, documentos de trabajo. 